\title{
Maternal Hypothyroxinemia During Pregnancy and Growth of the Fetal and Infant Head
}

Reproductive Sciences

19(I2) 1315-1322

(C) The Author(s) 2012

Reprints and permission:

sagepub.com/journalsPermissions.nav DOI: I0.1 I77/I9337|9| I 2450338

http://rs.sagepub.com

@SAGE

\author{
Nina H. van Mil, BMedSci ${ }^{1,2,3}$, \\ Régine P. M. Steegers-Theunissen, $M D, P^{2} D^{3,4,5}$, \\ Jacoba J. Bongers-Schokking, MD, PhD ${ }^{6}$, Hanan El Marroun, PhD ${ }^{1,2}$, \\ Akhgar Ghassabian, MD ${ }^{1,2}$, Albert Hofman, MD, PhD ${ }^{1,4}$, \\ Vincent W. V. Jaddoe, MD, PhD ${ }^{1,4,7}$, Theo J. Visser, PhD $^{8}$, \\ Frank C. Verhulst, MD, PhD ${ }^{2}$, Yolanda B. de Rijke, PhD $^{8,9}$, \\ Eric A. P. Steegers, MD, PhD $^{3}$, and \\ Henning Tiemeier, MD, $\mathrm{PhD}^{2,4,10}$
}

\begin{abstract}
Severe maternal thyroid dysfunction during pregnancy affects fetal brain growth and corticogenesis. This study focused on the effect of maternal hypothyroxinemia during early pregnancy on growth of the fetal and infant head. In a population-based birth cohort, we assessed thyroid status in early pregnancy (median 13.4, 90\% range 10.8-17.2), in 4894 women, and measured the prenatal and postnatal head size of their children at 5 time points. Hypothyroxinemia was defined as normal thyroidstimulating hormone levels and free thyroxine-4 concentrations below the 10th percentile. Statistical analysis was performed using linear generalized estimating equation. Maternal hypothyroxinemia was associated with larger fetal and infant head size (overall estimate $\beta: 1.38,95 \%$ confidence interval $0.56 ; 2.19, P=.001$ ). In conclusion, in the general population, even small variations in maternal thyroid function during pregnancy may affect the developing head of the young child.
\end{abstract}

\section{Keywords}

thyroid hormones, human fetuses, ultrasound, population-based cohort

\section{Introduction}

Thyroid hormones are important for different developing organ systems such as the kidney, lung, and skeleton. Furthermore, thyroid hormones play a crucial role, particularly in fetal brain maturation. ${ }^{1}$ As supported by experimental data in animals, a shortage of maternal thyroid hormones impacts the cytoarchitecture and radial distribution of specific neurons in the somotosensory cortex and hippocampus of the fetus. ${ }^{2,3}$ Other studies have suggested a detrimental effect of maternal thyroid dysfunction in pregnancy on the cognitive and behavioral development of the child. ${ }^{4}$ Recently, we found that a low maternal free thyroxine-4 (FT4) concentration in early pregnancy predicts the cognitive delay in children. ${ }^{5}$

It has been shown that children with congenital hypothyroidism have larger head circumferences. ${ }^{6-9}$ Newborns of hypothyroid mothers are reported to have a smaller head circumference and lower birth weight. ${ }^{10}$ Impaired prenatal brain development, as indexed by fetal head growth may underlie this association. The consequences of subtle nonclinical variations in the maternal thyroid status during pregnancy on fetal

\footnotetext{
I The Generation R Study Group, Erasmus MC, University Medical Center Rotterdam, Rotterdam, Netherlands

${ }^{2}$ Department of Child and Adolescent Psychiatry/Psychology, Erasmus MC, University Medical Center Rotterdam, Rotterdam, Netherlands

${ }^{3}$ Department of Obstetrics \& Gynecology, Erasmus MC, University Medical Center Rotterdam, Rotterdam, Netherlands

${ }^{4}$ Department of Epidemiology, Erasmus MC, University Medical Center Rotterdam, Rotterdam, Netherlands

${ }^{5}$ Department of Clinical Genetics, Erasmus MC, University Medical Center Rotterdam, Rotterdam, Netherlands

${ }^{6}$ Department of Endocrinology, Erasmus MC, University Medical Center Rotterdam, Rotterdam, Netherlands

${ }^{7}$ Department of Paediatrics, Erasmus MC, University Medical Center Rotterdam, Rotterdam, Netherlands

${ }^{8}$ Department of Internal Medicine, Erasmus MC, University Medical Center Rotterdam, Rotterdam, Netherlands

${ }^{9}$ Clinical Chemistry, Erasmus MC, University Medical Center Rotterdam, Rotterdam, Netherlands

${ }^{10}$ Department of Psychiatry, Erasmus MC, University Medical Center Rotterdam, Rotterdam, Netherlands
}

\section{Corresponding Author:}

Henning Tiemeier, Department of Child and Adolescent Psychiatry, Erasmus Medical Center, Sophia Children's Hospital, University Medical Center Rotterdam, P.O. Box 2060, 3000 CB Rotterdam, Netherlands

Email: h.tiemeier@erasmusmc.nl 
head and brain development, however, are not clear. Moreover, birth outcomes such as birth weight or head circumference at birth are only crude summary measures of intrauterine growth and cannot provide information of the growth across different time periods in pregnancy. Neonates can reach the same birth weight by different fetal growth trajectories. ${ }^{11}$ Studies that investigate the influence of maternal thyroid status on fetal head growth during pregnancy are scarce. ${ }^{12}$

Therefore, in the current study, we investigated whether maternal thyroid status assessed in early pregnancy is related to prenatal and postnatal head growth. We also explored whether any effect on the growth trajectories of the fetal head mediates the association between maternal thyroid function and postnatal cognitive infant development.

\section{Materials and Methods}

\section{Design and Study Population}

This study was embedded in the Generation R Study, an ongoing population-based birth cohort from fetal life onward. The Generation R Study, designed to identify early environmental and genetic determinants of growth, development and health, has been previously described in detail. ${ }^{13}$ Assessments were performed with ultrasonography, physical examinations, biological samples, and detailed questionnaires.

In mothers of 5831 fetuses, thyroid-stimulating hormone (TSH) and FT4 concentrations were measured during early pregnancy. Twin pregnancies $(\mathrm{n}=124)$ were excluded since maternal thyroid function and growth potentials for individual fetuses in multiple pregnancies are different from singleton pregnancies. We excluded pregnancies of mothers receiving any thyroid-related medication including thyroxin $(n=39)$ leaving 5276 children. Totally, 392 mothers participated with 2 or 3 siblings. Since the results did not differ after random exclusion of 1 or 2 of the siblings, they were included in the analyses. We excluded 256 mothers with thyroid problems other than hypothyroxinemia. Of the remainder, 4638 mothers had at least 1 prenatal or postnatal measurement of head circumference and were included in one or more analyses.

The study has been approved by the Medical Ethics Committee of the Erasmus Medical Center, Rotterdam. Written informed consent was obtained from all participants before participation.

\section{Assessment of Maternal Thyroid Function and Urinary lodine}

In early pregnancy (median 13.4, 90\% range 10.8-17.2), venous blood samples were drawn in plain tubes. Subsequently, serum was transported to the regional laboratory for storage at $-80^{\circ} \mathrm{C}$ within 3 hours after sampling. ${ }^{14}$ Maternal TSH and FT4 from the stored samples were assayed in batches of 50 to 150 over a 6-month period using a chemoluminescence assay on the Vitros ECI Immunodiagnostic System (ORTHO Clinical Diagnostics, Rochester, New York). The interassay coefficients of variation (CVs) for TSH and FT4 were $<4.1 \%$ and $<5.4 \%$, respectively, whereas the intraassay $\mathrm{CVs}$ were $<1.2 \%$ and $<2.7 \%$, respectively. Thyroid peroxidase (TPO) antibodies are measured using ImmunoCAP 250-assays (Phadia AB, Uppsala, Sweden). Maternal TPO-antibodies was measured using the Phadia 250 immunoassay (Phadia $\mathrm{AB}$ ) and regarded as positive when $>60 \mathrm{IU} / \mathrm{mL}$.

Reference ranges for maternal TSH (0.03-4.56 mIU/L), FT4 $(10.36-22.16 \mathrm{pmol} / \mathrm{L})$ levels were defined as the range between the 2.5th and 97.5th percentiles, after exclusion of women with known thyroid disease, thyroid-related medication usage, and twin pregnancies. Hypothyroxinemia was defined as normal TSH levels and FT4 concentrations below the 10th percentile $(11.82 \mathrm{pmol} / \mathrm{L})$. This definition has been applied to the current sample before. ${ }^{5}$

Maternal subclinical hypothyroidism (TSH $>97.5 \%$ and FT4 $>2.5 \% ; \mathrm{n}=31$ ), overt hypothyroidism (TSH $>97.5 \%$ and FT4 $<2.5 \% ; \mathrm{n}=5)$, and hyperthyroidism $(\mathrm{TSH}<2.5 \%$ and FT4 $>97.5 \% ; n=14$ ) were not studied as determinant due to the small number of children at risk.

At the same time of blood sampling, maternal single voided urine samples were collected at random moments over the day. In a subsample of 1082 women, urinary iodine was measured through the ceri-arsenite reaction following destruction by means of ammoniumpersulfate. After brief centrifugation, sodium arsenite solution $(0.1 \mathrm{~mol} / \mathrm{L}$ in $1 \mathrm{~mol} / \mathrm{L}$ of sulfuric acid) was added. Subsequently, ceriammonium sulfate was added and color was allowed to develop at $250^{\circ} \mathrm{C}$ during 60 minutes. Optical density was assessed at $405 \mathrm{~nm}$. At a level of $1.7 \mu \mathrm{mol} / \mathrm{L}$ the within-assay $\mathrm{CV}$ was $5.1 \%$ and the between-assay CV was $14.3 \%$.

To adjust for total urinary volume, we used the iodine to creatinine ratio.

\section{Fetal Ultrasound Examinations and Postnatal Assessments}

Sonographers carried out fetal ultrasound examinations at the visits to the research centers in early, mid, and late pregnancy. Most assessments (88\%) were performed at the Generation R research center in Rotterdam. The remaining assessments were carried out in 1 of 5 collaborating hospitals. The fetal ultrasound examinations were used to establish gestational age and to assess the fetal growth characteristics. Crown-rump length was used for pregnancy dating until a gestational age of 12 weeks. The measurement of the biparietal diameter in the first trimester was used for pregnancy dating after 12 weeks.

The median (90\% range) gestational age for the fetal ultrasound examinations in early, mid, and late pregnancy was 13.4 (10.8-17.2) weeks, 20.5 (19.1-22.6) weeks, and 30.3 (29.0$32.2)$ weeks, respectively. Postnatal measurement in early and mid-infancy was performed at $2.1(1.0-3.8)$ and $6.3(5.5-11.3)$ months of infant age, respectively.

To control for possible symmetric growth restriction, we corrected for abdominal circumference in additional analyses. 
Measurements used for the present study, including head circumference and abdominal circumference, were performed using standardized techniques. The head circumference was measured at the level of the biparietal diameter and represents the outer perimeter of the fetal skull. Sonographers were blinded to the thyroid status of the pregnant women.

The intraobserver and interobserver reliability of fetal biometry measurements in early pregnancy within the Generation R Study is good (intraclass correlation coefficients for head circumference 0.995 [intraobserver] and 0.988 [interobserver] and for biparietal diameter 0.995 [intraobserver] and 0.994 [interobserver] with CVs between $1.8 \%$ and $3.8 \%)^{15}$

Postnatal measurements of child head circumference were collected at community health centers as part of routine health care using standardised procedures of health centers in the Netherlands. ${ }^{16}$

\section{Cognitive Assessment}

Parent Report of Children Abilities (PARCA) was used as an indicator of cognitive development. The PARCA compromises 48 parent-reported items on the ability of their children to perform specific nonverbal cognitive tasks at age 30 months. A delay was defined as nonverbal cognitive scores below the 15th age- and gender-specific percentile. Previously, we showed a consistent association of maternal hypothyroxinemia with delayed cognitive functioning. ${ }^{11}$

\section{Covariates}

Information on maternal age, parity, national origin, education, prenatal smoking and alcohol use, folic acid use, and prenatal psychological problems was obtained by questionnaires during pregnancy. Educational level of the mother was assessed by the highest completed education and reclassified into 3 categories: primary school, secondary school, and higher education. National origin of the mother was based on the country of birth of the parents. Maternal prenatal smoking and alcohol use were classified as "no use," "use until pregnancy was confirmed," and "continued use during pregnancy." Height and weight were measured without shoes and heavy clothing; body mass index (BMI) was calculated from height and weight (weight/height ${ }^{2}$ ). At 20 weeks of pregnancy, we measured maternal prenatal psychological problems using the Brief Symptom Inventory. ${ }^{13}$ Child's gender, birth weight, Apgar scores 1 minute after birth, and the mode of delivery were derived from medical records completed by gynecologists and midwives. To define the gestational age at birth, we used the last menstrual period of the mother and the ultrasound examination at the first prenatal visit.

\section{Statistical Analysis}

Maternal thyroid status was a categorical determinant in our analyses. First, to examine the associations between maternal thyroid status during early pregnancy and fetal or infant head size at different ages, linear regression models (ANOVA) were used. To enable comparison, these analyses were restricted to children with all 3 prenatal ultrasounds and 2 postnatal measurements of mothers with hypothyroxinemia of reference thyroid status $(n=2621)$.

Linear generalized estimating equation (GEE) analyses ${ }^{14}$ were used to study the longitudinal effects of maternal hypothyroxinemia on fetal and child head size in all 4638 children. With GEE analyses, repeatedly measured head size were analyzed over time, taking correlations within the same participant into account. We calculated the overall effect of maternal hypothyroxinemia on head size throughout the longitudinal period.

Additionally, to test a dose-response relation with head growth in the lower tail of the FT4 distribution, $P$ for trends were calculated. Aimed on this, hypothyroxinemia was divided in 2 categories: FT4 levels within the 5th to 10th percentile and FT4 $<5$ th percentile.

All analyses were adjusted for maternal age, BMI, parity, education, national origin, smoking, alcohol use, prenatal psychological problems, child's gender, intrauterine position, and gestational age at ultrasound measurement. For postnatal analyses, age at birth and birth weight were also added as confounders to the model. The choice of potential confounders was determined a priori based on earlier literature. ${ }^{17,18}$ Further covariates were included in the analyses if the effect estimates of maternal thyroid function changed $(\geq 5 \%)$. Pregnancy complications and birth weight did not pass this threshold.

Furthermore, we explored whether the fetal head growth mediated any observed association between thyroid status and cognitive delay. Aimed on this, we first tested whether the thyroid status increased the risk of cognitive delay in the current sample of children. Next, we used linear regression models (ANOVA) to study if head circumference during pregnancy is related to nonverbal cognitive functioning. Finally, we tested possible mediation by studying the effect estimate change if head size is added to the model of thyroid status and cognitive delay.

\section{Results}

Characteristics of pregnant women and their children categorized by thyroid status are presented in Table 1 . Based on the criteria described above, $476 \quad(9.7 \%)$ mothers had hypothyroxinemia. Hypothyroxinemic women are older, less educated, more often of non-Dutch national origin, and more often smoking in pregnancy. Urinary iodine concentration did not differ among hypothyroxinemic and nonhypothyroxinemic mothers. Children born to women with hypothyroxinemia have higher birth weight.

Table 2 presents the association between hypothyroxinemia and fetal head size by gestational age. In early pregnancy, head circumference of fetuses of hypothyroxinemic mothers was $0.7 \mathrm{~mm}$ (95\% confidence interval [CI] 0.2-1.2) larger as compared to the reference group $(89.6 \mathrm{~mm}$; $95 \%$ CI 89.4-89.7, $P=$ $.008)$. Later in pregnancy, there were no significant differences in fetal head circumference.

Postnatal children born to hypothyroxinemic mothers had larger head circumference compared to mothers with reference 
Table I. Study Population by Maternal Thyroid Status (Total $n=4638)^{a}$

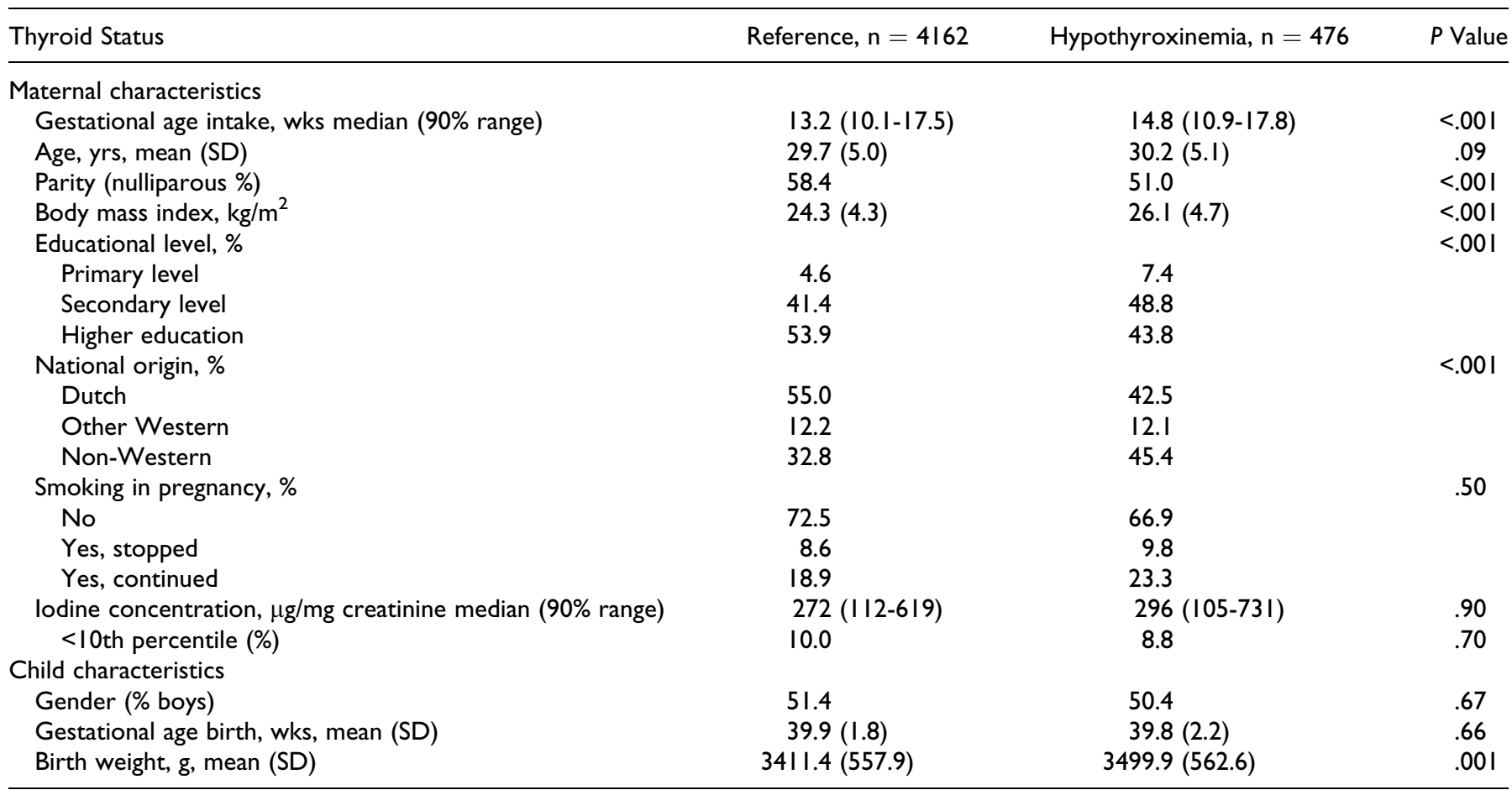

Abbreviation: SD, standard deviation.

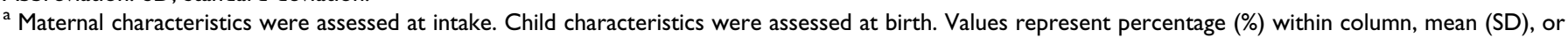
median ( $90 \%$ range). Independent Students $t$ test, Mann-Whitney $U$, and chi-square tested for differences in baseline.

Table 2. Fetal and Infant Head Size Stratified by Maternal Thyroid Status During Pregnancy ${ }^{\mathrm{a}}$

\begin{tabular}{|c|c|c|c|}
\hline \multicolumn{4}{|c|}{ Fetal and Infant Head Size in mm Stratified by Maternal Thyroid Status, N = 262I } \\
\hline Fetal head circumference & $\begin{array}{l}\text { Early pregnancy, } \\
\text { median I3.4 weeks }\end{array}$ & $\begin{array}{c}\text { Mid-pregnancy, } \\
\text { median } 20.5 \text { weeks }\end{array}$ & $\begin{array}{l}\text { Late pregnancy, } \\
\text { median } 30.3 \text { weeks }\end{array}$ \\
\hline Hypothyroxinemia, $\mathrm{mm}(95 \% \mathrm{Cl})$ & $90.3(89.8-90.8)$ & $179.1(178.4-179.9)$ & $286 . I(285.1-287.2)$ \\
\hline$P$ value for adjusted difference & .008 & .60 & .14 \\
\hline Infant head circumference & Early infancy, median 2.1 months & Mid-infancy, median 6.3 months & \\
\hline Hypothyroxinemia, mm (95\% Cl) & $394.3(393.0-395.6)$ & $440.9(439.4-442.3)$ & \\
\hline$P$ value for adjusted difference & .001 & .01 & \\
\hline
\end{tabular}

Abbreviation: $\mathrm{Cl}$, confidence interval.

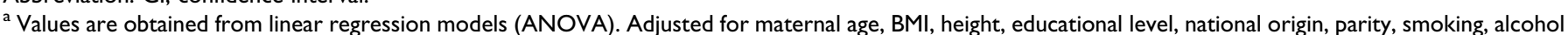

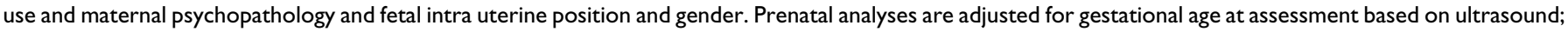
postnatal analyses are adjusted for infant age at assessment. Postnatal analyses are also adjusted for gestational age at birth.

thyroid status $(2.4 \mathrm{~mm} ; 95 \%$ CI $1.0-3.8, P=.001$ in early infancy and $2.0 \mathrm{~mm}$; 95\% CI 0.4-3.5, $P=.01$ in mid-infancy).

The GEE analysis, which summarizes the effect over time, showed a significant increased head size over time in children born to hypothyroxinemic mothers as compared to mothers with reference thyroid status ( $\beta$ : 1.38 , 95\% CI 0.56-2.19, $P=.001)$.

Figure 1 depicted the associations between hypothyroxinemia and head size at individual time points and during follow-up. The figure suggests that the effect of hypothyroxinemia on head size differs over time. Significant effects for individual time points in early pregnancy and infancy were observed, however, the differences between time points were not significant; indeed, the GEE model did not show any time differences $(\beta-.64,95 \% \mathrm{CI}-1.52-0.24, P=.16)$.

To distinguish between decreased head growth and a more general symmetrical growth restriction including decreased head circumference, we additionally adjusted for abdominal circumference measured at the same gestational age. The effect of hypothyroxinemia on fetal head circumference remained present (difference in head circumference for hypothyroxinemia early in pregnancy $0.7 \mathrm{~mm}, 95 \%$ CI $0.2-1.2, P=.01$ ). 


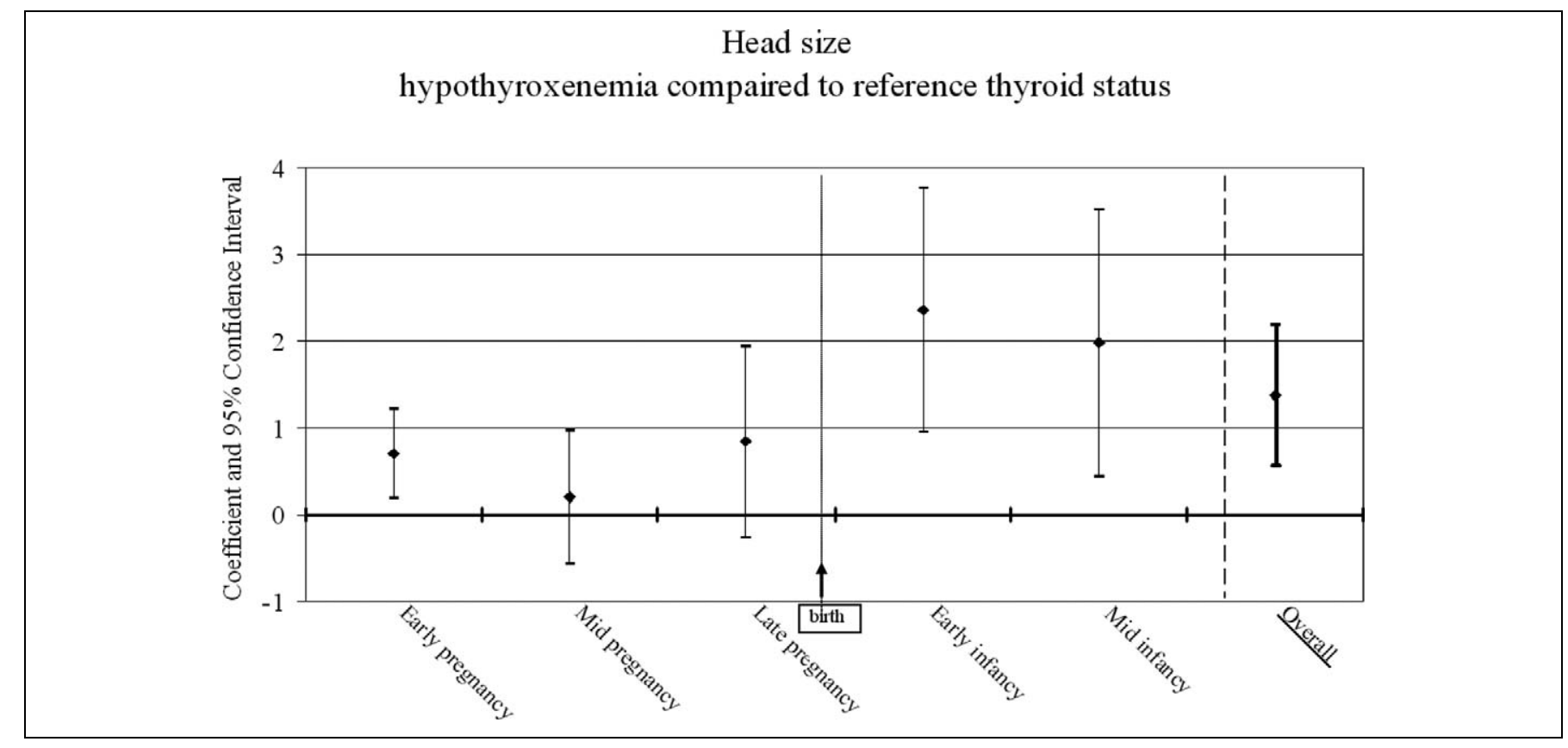

Figure I. Estimated differences in head circumference in fetuses or infants of mothers with hypothyroxinemia compared with the reference group as obtained from linear regression models. Generalized estimating equation (GEE) analysis was used to calculate the overall estimate. All models were adjusted for maternal age, body mass index (BMI), height, educational level, national origin, parity, folic acid supplement use, smoking, alcohol use and maternal psychological symptoms and child gender, intrauterine position, and (gestational) age at assessment. Postnatal and longitudinal analyses were also adjusted for gestational age at birth.

Next, we explored whether the effect of hypothyroxinemia was dose responsive. There was a dose-response relation in the lower range of the FT4 distribution between the degree of hypothyroxinemia and fetal head growth in early pregnancy $(\beta$ for linear contrast $0.64,95 \%$ CI $0.14-1.13, P$ for trend $=.01$ ).

Finally, we tested whether head size early in pregnancy mediated the relation between maternal thyroid status and cognitive delay. In the current sample, hypothyroxinemia was associated with an increased risk of cognitive delay (odds ratio $[\mathrm{OR}]=1.63,95 \%$ CI 1.06-2.52, $P=.03$ ). Head circumference measured during early pregnancy showed no association with cognitive delay ( $\beta-0.007,95 \% \mathrm{CI}-0.04-0.03, P=.99)$. This means that children with larger heads, early in pregnancy, did not have a higher risk of cognitive delay. The relation between thyroid status and cognitive delay can thus not be explained by early fetal head size. Using continuous measures of cognitive delay or curvilinear models of head size did not provide any evidence for mediation by fetal head size either (data not shown).

\section{Discussion}

In our study, maternal hypothyroxinemia in early pregnancy was associated with a larger size of the fetal and infant head. In early pregnancy, the effect was dose-response; severe hypothyroxinemia was associated with more severe deviations in head growth than mild hypothyroxinemia.

The growth of the fetal head did not primarily differ due to fetal dating differences in early pregnancy. In principle, the observed effects could reflect differences in fetal dating in early pregnancy. This seems unlikely since both sonographers and patients were blinded to thyroid status and hypothyroxinemia is an asymptomatic condition. The observed growth difference showed a consistent dose-response pattern in early pregnancy and the difference in head size continued after birth. In our study, hypothyroxinemia was not due to lower iodine concentrations suggesting that thyroid problems in this iodine sufficient area mostly have other origins. However, iodine excretion was measured only in a subsample.

Different explanations may help to understand the untoward effects of low FT4 levels in pregnancy on prenatal and postnatal head growth.

First, fetal growth may be impaired by low FT4 levels that affect placentation in early pregnancy. This is supported by the associations between maternal thyroid dysfunction and pregnancy complications related to malplacentation, including preeclampsia, intrauterine growth retardation, and still birth. It has been suggested that uteroplacental tissues are sensitive to minor perturbations in maternal thyroid function. ${ }^{19}$ A relation between placental insufficiency and a smaller head size in childhood with increasing divergence after puberty has been reported..$^{20}$ On the other hand, our study also showed reduced growth of the fetal head circumference, even after controlling for the effects of low maternal FT4 levels on abdominal circumference. This indicates that thyroid hormones have a differential effect of on fetal head size.

A second explanation for the effect of the prenatal maternal hypothyroxinemia is the direct effect of thyroid hormones on 
the developing brain. Such a mechanism has mainly been shown in animal studies. Ausó et al reported that FT4 deficits in rats alter normal migration of cortical neurons and the cytoarchitectonic organization of the neocortex and hippocampus in the offspring. ${ }^{2}$ If we infer the critical period from animal studies, the human fetal brain should be most vulnerable to maternal hypothyroxinemia in late first trimester or midgestation. In that period, neurogenesis, myelination, and apoptosis take place that might explain the observed differences in size in early pregnancy. ${ }^{21,22}$ Around birth synaptogenesis starts and the number of synapses growths exponential till the first year of life. ${ }^{23}$ Synaptogenesis starts to accelerate at the beginning of the third trimester of pregnancy and increases rapidly in the first 2 years of life. However, we argue as no statistically significant differences were observed between time points this interpretation is very speculative.

Apoptosis is important for normal brain development as $20 \%$ to $50 \%$ of neurons are known to undergo programmed cell death. ${ }^{24}$ In rats, neonatal administration of thyroxin produces smaller cerebellum with fewer folia. ${ }^{21,22}$ Studies on the in vivo effects of thyroid hormone upon neurons of the cerebral cortex show that thyroid hormone produces an increase of proteasome-ubiquitin-protein conjugates and an increase in apoptosis. ${ }^{25,26}$ This implies that a decrease in thyroxin need not lead to a decrease in brain tissue.

Third, as animal studies have shown, maternal thyroid hormones may affect fetal programming by epigenetic mechanisms in the structures and functions of organs. This is substantiated by studies showing acute changes in the maternal thyroid hormones that directly affect the expression of selected and stage-specific genes in the fetal brain. ${ }^{27,28} \mathrm{~A}$ decrease in T4 resulted in less expression of neuroendocrine-specific protein. This protein inhibits axonal growth action of central myelin in growth cone collapse and neurite outgrowth ${ }^{29}$ that probably results in increased brain mass. Again, this is in line with our observation that lower thyroid levels in early life are associated with a larger head circumference.

A fourth explanation for our observation might be that a lack of thyrotropin caused abnormal skull development, and abnormalities in thyrotropin metabolism are known to affect the skeletal development. However, the described delay in ossification and reduction of bone mineralization typically decrease rather than increase growth velocity. ${ }^{30}$ Furthermore, experimental work has shown that the growth of the vault of the skull depends on and is controlled by the growth of the brain. ${ }^{31}$

As head circumference is known to correlate to brain volume, it is tempting to speculate that increased head growth is one of the mediators in the relationship between maternal thyroid status in pregnancy and psychological and behavioral child development. ${ }^{32}$ However, children with autism spectrum disorders also show increased growth in head circumference during postnatal life. While there are probably some differences in the brain growth trajectories between these 2 conditions (ie, the exact onset of altered head growth in children with autism, ${ }^{33}$ it is tempting to speculate that related neurobiological mechanisms, such as the postnatal acceleration of formation of cortical neurones, ${ }^{34,35}$ underlie both conditions.
Besides, data from the present cohort showed that maternal hypothyroxinemia negatively affected behavior and cognitive functioning in infants. ${ }^{5,36}$ Inverted U-shaped associations between mid and late pregnancy head size and infant alertness have been reported previously indicating that both smaller and larger heads are negatively associated with this indicator of early neurobehavioral development. ${ }^{37}$ However, here we found no indication that a larger head in the first trimester is related to cognitive functioning. Possibly, structural changes not expressed by changes in head size may underlie this cognitive delay. Alternatively, a large head can reflect healthy and untoward effects, and this covers the negative impact of maternal thyroid status. Second, a catch-up and compensatory effect of brain development may make any early pregnancy effect not observable in toddlers anymore.

The strengths of this present study include our combination of ultrasound and postnatal measurements, which enabled us to determine the growth trajectories throughout gestation and infancy.

The large population-based prospective cohort enabled us to control for important confounding factors, including lifestyle factors, socioeconomic factors, and known determinants of fetal and infants' growth. Despite this, it cannot be ruled out that the associations are confounded by diet-related determinants or other unknown factors. Unfortunately, postnatal head circumference was not measured routinely at the Dutch child health care centres after the first year anymore. Longer follow-up studies are necessary to determine whether maternal hypothyroxinemia affects child head growth later in life. Finally, since data were more complete in higher-educated mothers, we cannot rule out that selective nonresponse influenced our findings.

In conclusion, in the general population, even small variations in maternal thyroid function during pregnancy may affect the developing head of the young child.

Low maternal FT4 increases the head circumference in the first trimester of pregnancy and in early infancy. However, the effect of altered head growth on cognitive functioning is not yet clear. Further studies of the possible effect of screening and intervention based on maternal thyroid function are needed.

\section{Acknowledgments}

The Generation R Study is conducted by the Erasmus Medical Center Rotterdam in close collaboration with the Faculty of Social Sciences of the Erasmus University Rotterdam, the Municipal Health Service Rotterdam area, the Rotterdam Homecare Foundation and the Stichting Trombosedienst \& Artsenlaboratorium Rijnmond (STAR), Rotterdam. We gratefully acknowledge the contribution of all participants, general practitioners, hospitals, midwives, and pharmacies in Rotterdam. We would like to acknowledge Mrs T. White, MD, PhD, for her insightful suggestions.

\section{Declaration of Conflicting Interests}

The authors declared no potential conflicts of interest with respect to the research, authorship, and/or publication of this article. 


\section{Funding}

The authors disclosed receipt of the following financial support for the research, authorship, and/or publication of this article: the general design of the Generation $\mathrm{R}$ Study was made possible by financial support from the Erasmus Medical Center, Rotterdam; the Erasmus University Rotterdam; the Dutch Ministry of Health, Welfare and Sport; and the Netherlands Organization for Health Research and Development (ZonMw). The current study was supported by a grant from ZonMw (grant no. 10.000.1003) and a research grant from the European Community's 7th Framework Programme (FP7/2008-2013) under grant agreement 212652 (NUTRIMENTHE project, "The Effect of Diet on the Mental Performance of Children"). NM was supported by an additional grant from the Sophia Foundation for Medical Research (SSWO grant number 268). The work of HT was supported by a NWO-ZonMW VIDI grant (grant no. 017.106.370).

\section{References}

1. Kester MH, Martinez de Mena R, Obregon MJ, et al. Iodothyronine levels in the human developing brain: major regulatory roles of iodothyronine deiodinases in different areas. J Clin Endocrinol Metab. 2004;89(7):3117-3128.

2. Auso E, Lavado-Autric R, Cuevas E, Del Rey FE, Morreale De Escobar G, Berbel P. A moderate and transient deficiency of maternal thyroid function at the beginning of fetal neocorticogenesis alters neuronal migration. Endocrinology. 2004;145(9): 4037-4047.

3. de Escobar GM, Ares S, Berbel P, Obregon MJ, del Rey FE. The changing role of maternal thyroid hormone in fetal brain development. Semin Perinatol. 2008;32(6):380-386.

4. Pop VJ, Brouwers EP, Vader HL, Vulsma T, van Baar AL, de Vijlder JJ. Maternal hypothyroxinaemia during early pregnancy and subsequent child development: a 3-year follow-up study. Clin Endocrinol (Oxf). 2003;59(3):282-288.

5. Henrichs J, Bongers-Schokking JJ, Schenk JJ, et al. Maternal thyroid function during early pregnancy and cognitive functioning in early childhood: the generation R study. J Clin Endocrinol Metab. 2010;95(9):4227-4234.

6. Ng SM, Wong SC, Didi M. Head circumference and linear growth during the first 3 years in treated congenital hypothyroidism in relation to aetiology and initial biochemical severity. Clin Endocrinol (Oxf). 2004;61(1):155-159.

7. Burt L, Kulin HE. Head circumference in children with short stature secondary to primary hypothyroidism. Pediatrics. 1977; 59(4):628-630.

8. Bucher H, Prader A, Illig R. Head circumference, height, bone age and weight in 103 children with congenital hypothyroidism before and during thyroid hormone replacement. Helv Paediatr Acta. 1985;40(4):305-316.

9. Grant DB. Growth in early treated congenital hypothyroidism. Arch Dis Child. 1994;70(6):464-458.

10. Blazer S, Moreh-Waterman Y, Miller-Lotan R, Tamir A, Hochberg Z. Maternal hypothyroidism may affect fetal growth and neonatal thyroid function. Obstet Gynecol. 2003;102(2):232-241.

11. Bloomfield FH, Oliver MH, Harding JE. The late effects of fetal growth patterns. Arch Dis Child Fetal Neonatal Ed. 2006;91(4): F299-F304.
12. Shields BM, Knight BA, Hill A, Hattersley AT, Vaidya B. Fetal thyroid hormone level at birth is associated with fetal growth. J Clin Endocrinol Metab. 2011;96(6):E934-E938.

13. De Beurs E. Brief Symptom Inventory, handleiding [Dutch manual]. Leiden, The Netherlands; 2004.

14. Diggle PJ, Liang KY, Zeger SL. Analysis of Longitudinal Data. Oxford, England: Oxford University Press; 2000.

15. Verburg BO, Mulder PG, Hofman A, Jaddoe VW, Witteman JC, Steegers EA. Intra- and interobserver reproducibility study of early fetal growth parameters. Prenat Diagn. 2008;28(4): 323-331.

16. Jaddoe VW, van Duijn CM, van der Heijden AJ, et al. The Generation R Study: design and cohort update 2010. Eur J Epidemiol. 2010;25(11):823-841.

17. Kramer MS. Intrauterine growth and gestational duration determinants. Pediatrics. 1987;80(4):502-511.

18. Mook-Kanamori DO, Steegers EA, Eilers PH, Raat H, Hofman A, Jaddoe VW. Risk factors and outcomes associated with first-trimester fetal growth restriction. JAMA. 2010;303(6): 527-534.

19. Cleary-Goldman J, Malone FD, Lambert-Messerlian G, et al. Maternal thyroid hypofunction and pregnancy outcome. Obstet Gynecol. 2008;112(1):85-92.

20. Pryor J, Silva PA, Brooke M. Growth, development and behaviour in adolescents born small-for-gestational-age. J Paediatr Child Health. 1995;31(5):403-407.

21. Benno R, Desroches D, Hahn M, Salinas J. Brain growth and behavior in developing Fuller BWS mice: effects of thyroid hormone. Soc Neurosci Abstr. 1985;11:529.

22. Lauder JM, Altman J, Drebs H. Some mechanisms of cerebellar foliation: effects of early hypo- and hyperthyroidism. Brain Res. 1974;76(1):33-40.

23. Thompson RA, Nelson CA. Developmental science and the media. Early brain development. Am Psychol. 2001;56(1):5-15.

24. Oppenheim RW. Cell death during development of the nervous system. Annu Rev Neurosci. 1991;14:453-501.

25. Pasquini LA, Marta CB, Adamo AM, Pasquini JM, Soto EF. Relationship between the ubiquitin-dependent pathway and apoptosis in different cells of the central nervous system: effect of thyroid hormones. Neurochem Res. 2000;25(5):627-635.

26. Marta CB, Adamo AM, Soto EF, Pasquini JM. Sustained neonatal hyperthyroidism in the rat affects myelination in the central nervous system. J Neurosci Res. 1998;53(2):251-259.

27. Dowling AL, Martz GU, Leonard JL, Zoeller RT. Acute changes in maternal thyroid hormone induce rapid and transient changes in gene expression in fetal rat brain. J Neurosci. 2000;20(6): 2255-2265.

28. Darras VM, Van Herck SL, Geysens S, Reyns GE. Involvement of thyroid hormones in chicken embryonic brain development. Gen Comp Endocrinol. 2009;163(1-2):58-62.

29. Woolf CJ. No Nogo: now where to go? Neuron. 2003;38(2): 153-156.

30. Bassett JH, Williams AJ, Murphy E, et al. A lack of thyroid hormones rather than excess thyrotropin causes abnormal skeletal development in hypothyroidism. Mol Endocrinol. 2008;22(2): 501-512. 
31. Tanner JM. Foetus Into Man: Physical Growth From Conception to Maturity. Cambridge, MA: Harvard University Press; 1990.

32. Cooke RW, Lucas A, Yudkin PL, Pryse-Davies J. Head circumference as an index of brain weight in the fetus and newborn. Early Hum Dev. 1977;1(2):145-149.

33. Courchesne E, Carper R, Akshoomoff N. Evidence of brain overgrowth in the first year of life in autism. JAMA. 2003;290(3):337-344.

34. Courchesne E, Mouton PR, Calhoun ME, et al. Neuron number and size in prefrontal cortex of children with autism. JAMA. 2011;306(18):2001-2010.
35. Schapiro S. Some physiological, biochemical, and behavioral consequences of neonatal hormone administration: cortisol and thyroxine. Gen Comp Endocrinol. 1968;10(2):214-228.

36. Ghassabian A, Bongers-Schokking JJ, Henrichs J, et al. Maternal thyroid function during pregnancy and behavioral problems in the offspring: the generation R study. Pediatr Res. 2011;69(5 Pt 1): 454-459.

37. Henrichs J, Schenk JJ, Schmidt HG, et al. Fetal size in mid- and late pregnancy is related to infant alertness: the generation $\mathrm{R}$ study. Dev Psychobiol. 2009;51(2):119-130. 